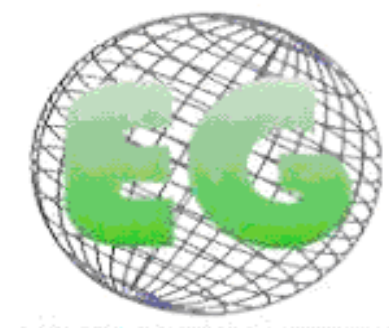

ISSN 1695-6141 $N^{\circ} 26$

\title{
Plan de cuidados estándar de enfermería en artroplastia de cadera
}

Standard plan nursing care in hip arthroplasty

*Bustos López, R., **Piña Martínez, AJ., **Pastor Molina, M., **Benítez Laserna, A. **Víllora Fernández, MP., ***González Pérez, AD.

*Lcdo. en Biología. DUE. Unidad de Enfermería de Traumatología. E.mail: rblcz@ono.com **DUE. Unidad de Enfermería de Traumatología. ***Médico residente $4^{\circ}$ año. Unidad de Cirugía Ortopédica y Traumatológica. Complejo Hospitalario Universitario de Albacete (CHUAB).

Palabras clave: plan de cuidados; metodología enfermera; artroplastia de cadera. Keywords: plan of care; nursing methodology; hip arthroplasty

\section{RESUMEN}

Los problemas derivados de una intervención de artroplastia de cadera afectan a prácticamente todos los patrones funcionales del paciente (Marjory Gordon), de ahí la importancia de que el personal sanitario implicado en el tratamiento y recuperación del paciente desarrolle su trabajo según un PLAN claramente definido, estándar y evaluable.

Éste ha sido el objetivo principal del equipo de trabajo, sistematizar los cuidados para que al paciente no le afecten los problemas derivados de la variabilidad en su atención, recupere su movilidad e independencia lo antes posible y se eviten complicaciones postoperatorias, para lo cual se ha prestado especial atención en la implicación del propio paciente en su recuperación. Administrativamente, con un Plan de Cuidados se mejora el registro de todas las actividades de enfermería y se clarifican las responsabilidades de los miembros del equipo sanitario.

En lo que respecta a la atención de enfermería, esta regulación en los cuidados se ha realizado siguiendo la taxonomía NANDA-NOC-NIC, resultando un plan estándar de cuidados basado en los Patrones funcionales de Marjory Gordon, ya que los autores del presente Plan de atención creemos que éste es método muy fiable para realizar la valoración de un paciente, de la cual se derivarían los diagnósticos de enfermería adecuados y la evaluación continua del paciente.

Concluimos que la aplicación de este Plan de Cuidados implicaría una mejora en la atención al paciente y en la organización del trabajo en la unidad de hospitalización de cirugía ortopédica y traumatológica. La validez del presente plan sería de tres años. 


\section{ABSTRACT}

The problems resulting from an intervention in hip, affect practically all functional patterns of the patient (Marjory Gordon), hence the importance of health personnel involved in treatment and recovery of the patient performing their work to a clearly defined standard and graded plan.

This has been the main objective of the team work, to systematize care for the patient so that it does not affect the problems of variability in their care, recover their mobility and independence as soon as possible and to avoid postoperative complications, for which special attention is paid to involving patients in their own recovery. Administratively, a Care Plan will improve the registration of all nursing activities and clarify the responsibilities of health team members.

With regard to nursing care, this care regulation has been made following the NANDA-NOC-NIC taxonomy, resulting in a standard plan of care based on Marjory Gordon's functional patterns, since the authors believe this is a very reliable method for conducting an assessment of the state of the patient, which would derive in the appropriate nursing diagnosis and ongoing evaluation of the patient.

We conclude that the application of the Care Plan would mean an improvement in patient care and organization of work in the hospital units of trauma and orthopaedic surgery. The validity of this plan would be three years.

\section{INTRODUCCIÓN}

La implementación de los diagnósticos de enfermería implica una mejor planificación de los cuidados y una mejora en la comunicación entre diferentes miembros del personal, y de éstos con el paciente.

Un plan de cuidados estandarizado, también mejora la organización clínica al conjuntar la teoría y la práctica enfermera resultando una enfermería basada en la evidencia. En el presente plan de cuidados, hemos tratado de responder a las preguntas de Quién, Cuándo y Dónde se debe de aplicar una determinada actividad para clarificar la responsabilidad exacta de cada miembro del equipo de la unidad de hospitalización y, por lo tanto, para aunar criterios en la atención integral al paciente, considerado éste como un ser bio-psico-social ya que, además de atender sus necesidades más puramente fisiológicas, se ha puesto especial énfasis en sus necesidades psicológicas (ansiedad, conocimientos deficientes, etc.) y en la enseñanza postquirúrgica, tanto del paciente como del cuidador principal, para prevenir complicaciones domiciliarias.

La principal indicación para la artroplastia de cadera es dolor intenso, crónico, que no cede con tratamiento conservador y que suele ir acompañado de fracaso funcional de la articulación. Las causas principales para este tratamiento en la actualidad son:

- Artrosis.

- Necrosis avasculares de cabeza de fémur.

- Displasias de cadera.

- Complicaciones de fracturas de cuello fémur.

Los tipos de prótesis que se utilizan en este hospital como artroplastia total primaria de cadera son dos: Prótesis de cadera convencional y prótesis de cadera de superficie. Dentro de las prótesis de cadera convencional hay variabilidad en el tipo de prótesis y en el par de fricción. También pueden ser cementadas o no cementadas según la indicación.

En nuestro hospital se realiza una media de 175 intervenciones de artroplastia total de cadera primarias al año que cumplen con los criterios de GRD 818 (sustitución de cadera excepto por complicaciones) y GRD 817 (sustitución de cadera por complicaciones) ${ }^{(1)}$. El 
rango de edad puede ser muy variable, incluyéndose desde la tercera década de la vida hasta la séptima u octava década, e incluyendo tanto hombres como mujeres.

\section{METODOLOGÍA}

Para la elaboración del presente plan de cuidados estándar, hemos realizado la lectura crítica de bibliografía buscada en las bases de datos Medline, Cuidenplus y la Cochrane Plus en castellano, así como usando búsqueda libre en motores de búsqueda en Internet. También hemos consultado directamente las páginas web de asociaciones científicas relacionadas con la traumatología y/o metodología enfermera.

Los descriptores usados en la búsqueda son: Plan de cuidados, artroplastia de cadera, metodología enfermera.

El Proceso de atención de enfermería, ha sido confeccionado según la codificación de la taxonomía NANDA-NOC-NIC ${ }^{(2)(3)(4)(5)}$, con una valoración del paciente basada en los Patrones funcionales de Marjory Gordon ${ }^{(6)}$, al igual que la elección de los diagnósticos de enfermería que han sido clasificados según la Clasificación de Dominios de la NANDA (Taxonomía II). Para la valoración del paciente, hemos usado la hoja de "Valoración inicial de enfermería" (Anexo I). También se usará el impreso de "Valoración de enfermería al alta" (Anexo II).

\section{OBJETIVOS.}

Los objetivos generales de todo plan de cuidados estándar, deberían ser: ${ }^{(2)}$

- Sistematizar los cuidados que reciben los pacientes y dotar al profesional de enfermería de un instrumento que mejore la práctica profesional con el fin de prevenir posibles complicaciones, reducir costes económicos y conseguir una recuperación más temprana del paciente.

- Asignar la responsabilidad de los trabajos.

- Realizar un cuidado integral al paciente.

- Registrar todas las actividades de enfermería.

- Educar e implicar al paciente y familia en los cuidados básicos de su enfermedad.

Los objetivos específicos del presente plan de cuidados estándar de artroplastia de cadera, además de los anteriores, comprenderán:

- Incremento de la movilidad funcional del paciente.

- La disminución del dolor.

- La mejora en la apreciación de la calidad de vida.

- El aumento en la independencia en las actividades de la vida diaria (AVD).

- El retorno precoz a domicilio (4ํ día postoperatorio.)

- Cumplimiento de los criterios de resultados marcados en el plan de cuidados.

\section{VALORACIÓN DEL PLAN DE CUIDADOS ESTÁNDAR DE ENFERMERÍA EN ARTROPLASTIA DE CADERA.}

El departamento de calidad del CHUAB, sería el encargado de evaluar anualmente el grado de cumplimiento del Plan de cuidados por parte del personal adscrito al servicio de Traumatología, así como los factores condicionantes de esa situación, con el fin de averiguar 
la aceptación del Plan por parte del personal y las posibles sugerencias que dicho personal pudiera hacer. Gracias a la próxima implantación en el CHUAB de la estación clínica informática MAMBRINO XXI y la inclusión del plan de cuidados en dicha estación, la evaluación de dicho plan sería bastante factible. El presente Plan estándar de cuidados tiene una validez de tres años.

\section{Abreviaturas:}

- CHUAB: Complejo Hospitalario Universitario de Albacete.

- NANDA: Nort American Nursing Diagnosis Association.

- NOC: Nursing Outcomes Classification.

- NIC: Nursing Interventions Classification.

- ETIQ.: Etiqueta NOC

- INDIC.: Indicadores NOC.

- DUE: Diplomado Universitario de Enfermería.

- AE: Auxiliar de Enfermería.

- DAV: Dispositivos de Acceso Venoso.

- r/c: relacionado con...

- m/p: manifestado por...

- HBPM: Heparina de Bajo Peso Molecular.

- ACP: Analgesia Controlada por el Paciente.

- AVD: Actividades de la Vida Diaria. 


\section{PLAN DE CUIDADOS ESTANDAR DE ARTROPLASTIA DE CADERA}

\section{DOMINIO N²: NUTRICIÓN.}

\begin{tabular}{|c|c|c|c|c|}
\hline \multicolumn{3}{|c|}{ DIAGNÓSTICO } & \multicolumn{2}{|r|}{ uidos r/c intervención quirúrgica y/o pérdidas excesivas a } \\
\hline \multirow{3}{*}{ Z } & \multicolumn{2}{|c|}{$\frac{\text { ETIQUETA }}{\text { (ETIQ). }}$} & \multicolumn{2}{|c|}{$\begin{array}{l}\text { - (0601) Equilibrio hídrico (equidistrangio hídrico). } \\
\text { - (0401) Estado circulatorio. }\end{array}$} \\
\hline & \multirow{2}{*}{ 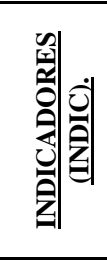 } & \multicolumn{2}{|r|}{$\begin{array}{l}\text { - (060116) Hidratación cutánea. } \\
\text { - (060107) Entradas y salidas diarias equilibradas. } \\
\text { - (060105) Pulsos periféricos. } \\
\text { - (060019) Ausencia de irritabilidad neuromuscular. }\end{array}$} & \begin{tabular}{lll} 
GRAVEMENTE & \multicolumn{2}{c}{ COMPROMISO } \\
1 & $\frac{\text { BASANTE }}{2} \frac{\text { MEDIO }}{3}$ & $\frac{\text { POCO }}{4}$
\end{tabular} \\
\hline & & \multicolumn{2}{|r|}{$\begin{array}{l}\text { - (040104) Presión arterial media. } \\
\text { - (080205) PA sistólica } \\
\text { - (080202) Frecuencia del pulso apical }\end{array}$} & 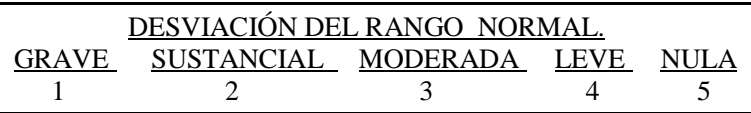 \\
\hline$=$ & \multicolumn{4}{|c|}{$\begin{array}{ll}\text { - (0601) Manejo de la hipovolemia. } & -(6 \\
\text { - (4030) Administración de productos sanguíneos. } & -(2 \\
\text { - (4160) Control de hemorragias. } & -(4 \\
\text { - (4010) Prevención de hemorragias. } & -(4 \\
\text { - (6650) Vigilancia. } & -(2 \\
\text { - (4190) Punción intravenosa } & \\
\end{array}$} \\
\hline 妾 & \multicolumn{4}{|c|}{$\begin{array}{l}\text { - El DUE tomará y registrará las constantes vitales del paciente a su llegada a planta y posteriormente cada } 8 \text { horas. (Tensión } \\
\text { arterial, frecuencia cardiaca). El AE tomará la temperatura del paciente a su llegada a planta y a requerimiento del DUE. } \\
\text { - El DUE administrará la fluidoterapia según tratamiento prescrito (Suero fisiológico o Ringer lactato } 500 \mathrm{cc} \text { c/6-8 h) y } \\
\text { aplicará la restricción de sueroterapia prescrita una vez el paciente haya tolerado dieta líquida. } \\
\text { - El AE tomará y registrará la diuresis y pérdidas por drenajes del paciente (en caso de portarlo) a su llegada a planta y } \\
\text { posteriormente cada } 12 \text { horas. } \\
\text { - El DUE vigilará el estado de piel y mucosas para valorar el estado de hidratación del paciente. } \\
\text { - El DUE vigilará ritmo de fluidoterapia y la zona de punción de la vía venosa según el protocolo de vías venosas del CHUA. } \\
\text { - El DUE vigilará tolerancia oral a líquidos del paciente a las } 6 \text { horas postintervención y posteriormente la tolerancia a sólidos. } \\
\text {-El DUE vigilará el estado del apósito para descartar posible hemorragia por herida quirúrgica. } \\
\text { - En el } 3^{\circ} \text { día, el DUE retirará vía venosa si el paciente no tiene medicación intravenosa. }\end{array}$} \\
\hline
\end{tabular}

DOMINIO Nº 3 : ELIMINACIÓN / INTERCAMBIO.

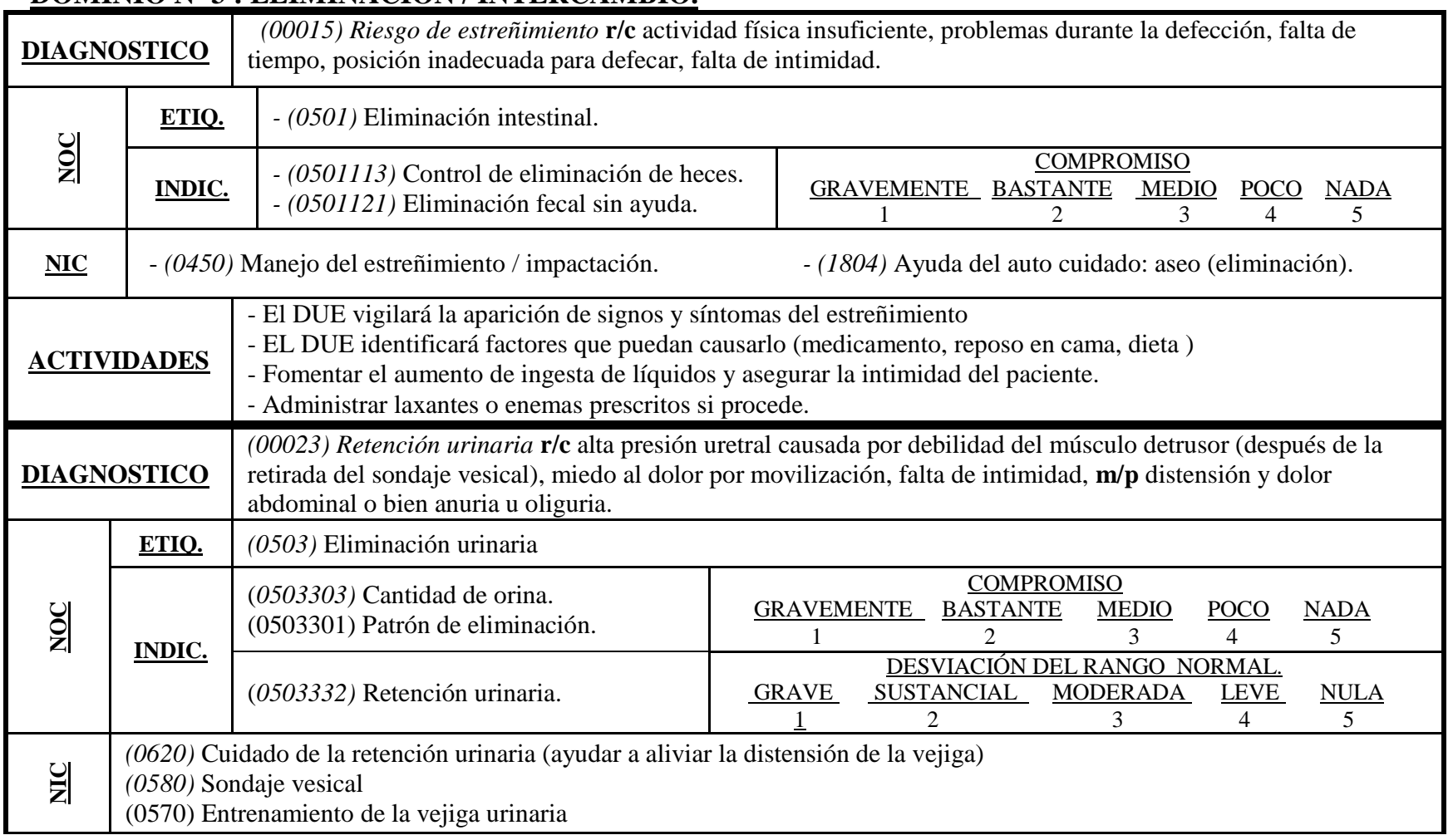


- Vigilar el grado de distensión de la vejiga y observar signos y síntomas de retención urinaria.

- Hacer maniobra de Credé : se hace presión sobre la vejiga para ayudar a eliminar orina

- Controlar la ingesta y líquidos

- Proporcionar intimidad en la habitación

- Poner sonda vesical, según protocolo del CHUAB, si precisa.

DOMINIO N 4 : ACTIVIDAD / REPOSO.

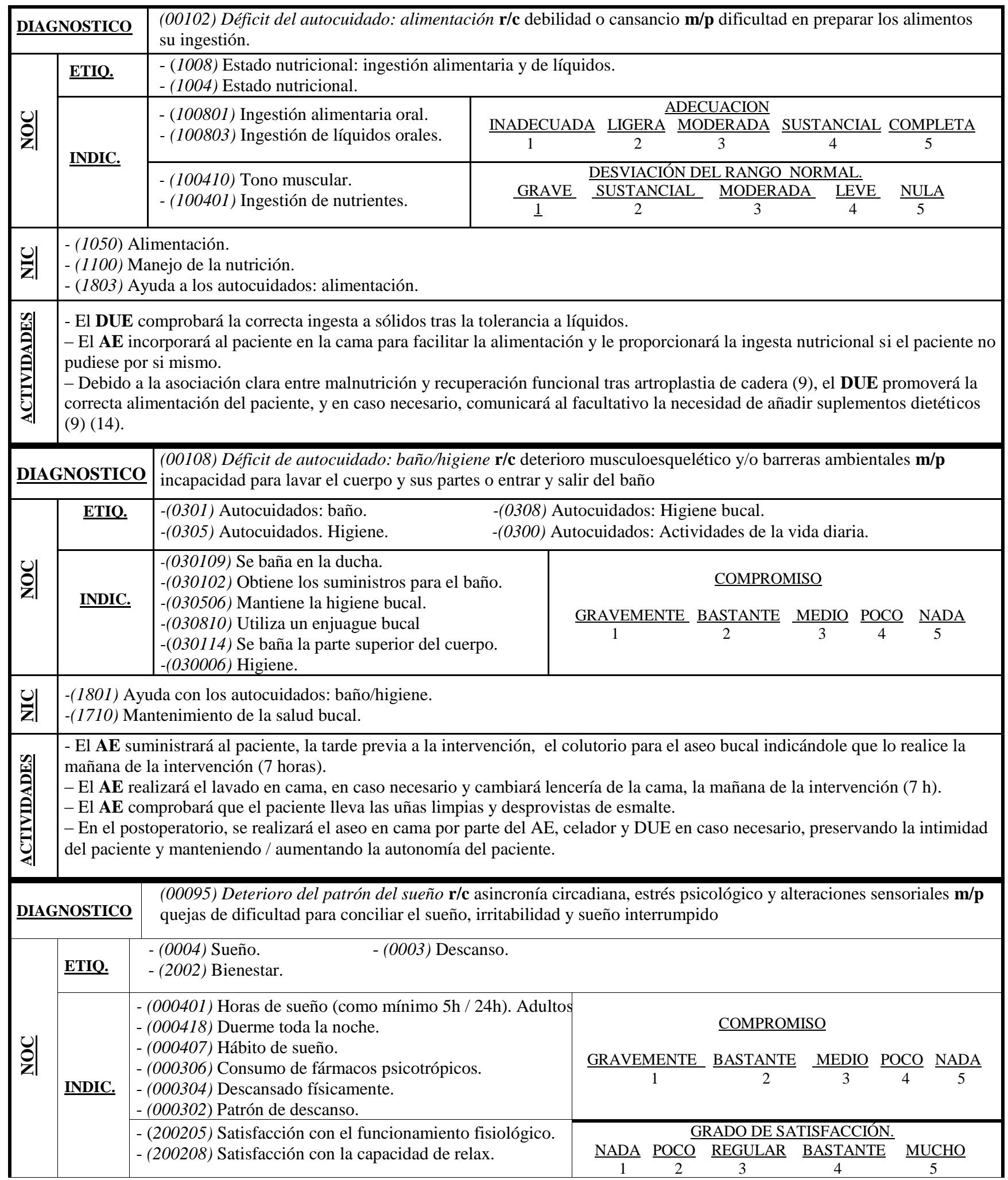




\begin{tabular}{|c|c|c|}
\hline$\vec{z}$ & $\begin{array}{l}\text { - (1850) Fomentar el sueño. } \\
\text { - (0180) Manejo de la energía. } \\
\text { - (6480) Manejo ambiental: confort. } \\
\text { - (6040) Terapia de relajación simple. }\end{array}$ & $\begin{array}{l}\text { - (2300) Administración de la medicación. } \\
\text { - (5380) Potenciación de la seguridad. } \\
\text { - (5230) Aumentar el afrontamiento. }\end{array}$ \\
\hline 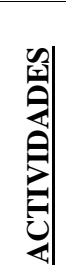 & $\begin{array}{l}\text { - El personal de la unidad fomentará } \\
\text { explicándole las ventajas de su nueva } \\
\text { fisiológicas de la zona intervenida (inf } \\
\text { - El personal de la unidad proporcio } \\
\text { minimizando el encendido de luces, re } \\
\text { etc... } \\
\text { - El DUE administrará la benzodiacep }\end{array}$ & $\begin{array}{l}\text { rcadiano evitando en lo posible el sueño diurno. Se tranquilizará al paciente } \\
\text { cadera (respecto a duración y seguridad), así como las normales reacciones } \\
\text { hematoma inguinal, pequeña hemorragia, etc). } \\
\text { biente tranquilo y relajado al paciente, sobretodo en horario nocturno, } \\
\text { or el altavoz de la habitación, administrando medicación antes de las } 24 \text { horas, } \\
\text { rita y vigilará su efectividad. }\end{array}$ \\
\hline
\end{tabular}

DOMINIO N' 4: ACTIVIDAD / REPOSO.

\begin{tabular}{|c|c|c|c|}
\hline \multicolumn{2}{|c|}{ DIAGNOSTICO } & \multicolumn{2}{|c|}{$\begin{array}{l}\text { (00085) Deterioro de la movilidad física } \mathbf{r} / \mathbf{c} \text { disminución de la fuerza, dolor, miedo a iniciar el movimiento } \mathbf{~ m} / \mathbf{p} \\
\text { dificultad para realizar las actividades del autocuidado, reacio a intentar el movimiento y disminución de la fuerza, } \\
\text { control y/o masa muscular. }\end{array}$} \\
\hline \multirow{4}{*}{ ○్ } & ETIQ. & $\begin{array}{l}\text { - (1811) Conocimiento: actividad preescrita. } \\
\text { - (0208) Nivel de movilidad } \\
\text { - (0200) Deambulación: Caminata. }\end{array}$ & $\begin{array}{l}\text { 0216) Movimiento articular. } \\
\text { 0201) Realización del traslado. }\end{array}$ \\
\hline & \multirow{3}{*}{$\underline{\text { INDIC. }}$} & $\begin{array}{l}\text { - (181104) Descripción de las restricciones de la } \\
\text { actividad. } \\
\text { - (181101) Descripción de la actividad prescrita. }\end{array}$ & \multirow{3}{*}{\begin{tabular}{|l}
$\frac{\text { NINGUNO }}{1} \frac{\text { ESCASO }}{2} \frac{\text { MODERADO }}{3} \frac{\text { SUSTANCIAL }}{4} \frac{\text { EXTENSO }}{5}$ \\
$\frac{\text { GRAVEMENTE }}{1} \frac{\frac{\text { COMPROMISO }}{\text { BASTANTE }}}{2} \frac{\text { MEDIO }}{3} \frac{\text { POCO }}{4} \frac{\text { NADA }}{5}$ \\
$\frac{\text { GRAVE }}{\frac{1}{2}} \frac{\text { SESVIACIÓN DEL RANGO NORMAL. }}{2} \frac{\text { MODERADA }}{3} \frac{\text { LEVE }}{4} \frac{\text { NULA }}{5}$
\end{tabular}} \\
\hline & & $\begin{array}{l}\text { - (020804) Movimiento articular. } \\
\text { - }(020803) \text { Movimiento muscular. } \\
\text { - }(021002) \text { Traslado de la silla a la cama. } \\
\text { - }(021001) \text { Traslado de la cama a la silla. } \\
\text { - }(020014) \text { Anda por la habitación. } \\
\text { - }(020002) \text { Camina con marcha eficaz. } \\
\text { - }(021105) \text { Alineación esquelética. } \\
\text { - (020809) Coordinación. } \\
\end{array}$ & \\
\hline & & $\begin{array}{l}\text { (021609/01) Flexión rodilla recta } 90^{\circ} \text { (izquierda / } \\
\text { derecha) } \\
(021610 / 02) \text { Extensión rodilla recta } 0^{\circ} \text { ( } \\
\text { izquierda/derecha) } \\
(021606 / 14) \text { Abducción de } 30^{\circ} \text { ( izquierda/derecha) } \\
(021616 / 08) \text { Rotación externa ( izquierda/derecha) }\end{array}$ & \\
\hline 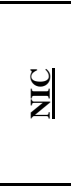 & \multicolumn{2}{|c|}{$\begin{array}{l}\text { - (0740) Cuidados del paciente encamado. } \\
\text { - (0202) Fomento del ejercicio: extensión. } \\
\text { - (0840) Cambio de posición. } \\
\text { - (0226) Terapia de ejercicios: control muscular } \\
\text { - (0140) Fomentar los mecanismos corporales. }\end{array}$} & $\begin{array}{l}\text { - (5612) Enseñanza actividad/ejercicio preescrito. } \\
\text { - (1400) Manejo del dolor. } \\
\text { - (0221) Terapia de ejercicios: ambulación. } \\
\text { - (0222) Terapia de ejercicios: equilibrio } \\
\text { - (0224) Terapia de ejercicios: movilidad articular. }\end{array}$ \\
\hline 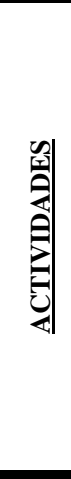 & \multicolumn{3}{|c|}{$\begin{array}{l}\text { - El DUE explicará al paciente y familiar las razones del reposo en cama. Se fijará el trapecio a la cama. } \\
\text { - El DUE colocará al paciente con alineación en cama decúbito supino y con las medidas antiaducción (almohada entre } \\
\text { miembros inferiores). (11) El DUE cursará radiografía de control prescrita por el facultativo. } \\
\text {-El DUE enseñará al paciente los ejercicios de flexión y extensión del miembro operado y fomentará su realización por parte } \\
\text { del paciente. Así mismo, el DUE administrará la profilaxis antitromboembólica preescrita, (HBPM } \mathbf{4 0} \mathbf{~ m g ~ S C ~ c / 2 4 ~ h . ~ G r a d o ~} \\
\text { de recomendación A) (12)(13). } \\
\text { - El AE y DUE mantendrán la ropa de la cama limpia, seca y sin arrugas, colocando el timbre y pulsador de la luz a su alcance. } \\
\text { - El paciente, tras la realización de radiografía de control, se levantará al sillón con ayuda moderada en la trasferencia. El DUE } \\
\text { comprobará que el paciente es sentado en un sillón de asiento alto, inmediatamente después de haber sido aseado y curado y } \\
\text { con la ayuda del AE y celador de la planta.. El } 3^{\circ} \text { día postoperatorio, la transferencia se realizará con ayuda mínima (14) y el } \\
\text { paciente deberá quedarse sentado un mínimo de } 2 \text { horas antes de empezar la deambulación. } \\
\text { - El DUE ayudará en la deambulación del paciente con andador durante } 8 \text { metros en el turno de mañana y tarde (14), } \\
\text { indicándole la forma correcta de hacerlo (andador, iniciando primero el miembro operado y después el otro).(11) }\end{array}$} \\
\hline \multicolumn{2}{|c|}{ DIAGNOSTICO } & \multicolumn{2}{|c|}{$\begin{array}{l}\text { (00091) Deterioro de la movilidad en la cama r/c prohibición de decúbito lateral debido a intervención quirúrgica } \\
\mathbf{m} / \mathbf{p} \text { restricciones impuestas por el movimiento. }\end{array}$} \\
\hline \multirow[b]{2}{*}{ Е| } & IIQ. & \multicolumn{2}{|l|}{-(0280) Nivel de movilidad. } \\
\hline & DIC. & $\begin{array}{l}\text { - (020804) Movimiento articular. } \\
\text { - (020803) Movimiento muscular. } \\
\text { - (020814) Se mueve con facilidad. }\end{array}$ & $\frac{\text { AVEMENTE }}{1} \frac{\text { COMPROMISO }}{2} \frac{\text { BASTANTE }}{2} \frac{\text { MEDIO }}{3}$ \\
\hline $\mathbf{z}$ & \multicolumn{3}{|c|}{$\begin{array}{l}\text { - (0740) Cuidados del paciente encamado. } \\
\text { - (0840) Cambios de posición. } \\
\text { - (1801) Ayuda con los autocuidados: baño/higiene. }\end{array}$} \\
\hline
\end{tabular}




\begin{tabular}{|l|l|}
\hline & - El DUE explicará al paciente y familiar las razones del reposo en cama. \\
- El DUE fomentará la comodidad, seguridad (barandillas laterales), y prevención de complicaciones del paciente inmóvil. \\
- El DUE informará y ayudará al paciente a girar sobre el miembro operado, nunca sobre el lado contralateral a la intervención \\
(riesgo de luxación de la prótesis). \\
- El AE ayudará al paciente en el aseo, le facilitará la cuña de eliminación explicándole su uso y poniéndosela en caso \\
necesario. \\
- El AE incorporará al paciente en la cama para facilitar la alimentación, le acercará la bandeja de comida y le dará de comer \\
en caso necesario.
\end{tabular}

\section{DOMINIO N 4: ACTIVIDAD / REPOSO. (BIS)}

\begin{tabular}{|c|c|c|c|}
\hline \multicolumn{2}{|c|}{ DIAGNOSTICO } & \multicolumn{2}{|c|}{$\begin{array}{l}\text { (00110) Déficit del autocuidado: uso del WC r/c trastorno de la capacidad de transferencia y/o dolor, molestias } \\
\mathbf{~ m / p ~ i n c a p a c i d a d ~ p a r a ~ i r ~ a l ~ b a n ̃ o ~ o ~ u s a r ~ e l ~ o r i n a l ~ o ~ s e n t a r s e ~ y ~ l e v a n t a r s e ~ d e l ~ i n o d o r o ~}\end{array}$} \\
\hline \multirow[b]{2}{*}{ ○्ञ } & ETIQ. & $\begin{array}{l}\text { - (0310) Autocuidados: Uso del inodoro } \\
\text { - (0305) Autocuidados. Higiene. }\end{array}$ & (0300) Autocuidados: actividades de la vida diaria. \\
\hline & $\underline{\text { INDIC. }}$ & $\begin{array}{l}\text { - (031008) Se levanta al inodoro. } \\
\text { - (030503) Se limpia la zona perineal. } \\
\text { - (030003) Uso del inodoro. }\end{array}$ & \begin{tabular}{lllll} 
GRAVEMENTE & \multicolumn{2}{c}{ COMPROMISO } \\
1 & $\frac{\text { BASTANTE }}{2}$ & $\frac{\text { MEDIO }}{3}$ & $\frac{\text { POCO }}{4}$ & $\frac{\text { NADA }}{5}$
\end{tabular} \\
\hline$\underline{\text { NIC }}$ & \multicolumn{2}{|c|}{$\begin{array}{l}\text { - (1804) Ayuda con los autocuidados: aseo (eliminación) } \\
\text { - (6480) Manejo ambiental }\end{array}$} & $\begin{array}{l}\text { - (0430) Manejo intestinal } \\
\text { - (0590) Manejo de la eliminación urinaria. }\end{array}$ \\
\hline \multicolumn{2}{|c|}{$\underline{\text { ACTIVIDADES }}$} & \multicolumn{2}{|c|}{$\begin{array}{l}\text { - La AE, proporcionará al paciente una cuña, explicará y ayudará en su uso, teniendo en cuenta la dificultad } \\
\text { para la movilidad y el dolor. } \\
\text { - Se deberá tener en cuenta la falta de intimidad y adecuar la situación (cortinas, numero de acompañantes, etc) } \\
\text { - En el } 3^{\circ} \text { día, se le suministrará al paciente un suplemento elevador del inodoro, explicándole su uso. }\end{array}$} \\
\hline \multicolumn{2}{|c|}{ DIAGNOSTICO } & \multicolumn{2}{|c|}{ (00094) Riesgo de intolerancia a la actividad $\mathbf{r} / \mathbf{c}$ inexperiencia en la actividad y mala forma física. } \\
\hline \multirow[b]{2}{*}{ 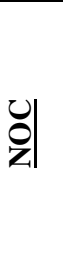 } & $\underline{\text { ETIQ. }}$ & $\begin{array}{l}\text { - (0002) Conservación de la energía. } \\
\text { - (0001) Resistencia. } \\
\end{array}$ & 005) Tolerancia de la actividad. \\
\hline & $\underline{\text { INDIC. }}$ & $\begin{array}{l}\text { - (000102) Actividad. } \\
\text { - (000118) Fatiga. } \\
\text { - (000518) Facilidad para realizar las AVD. } \\
\text { - (000509) Paso al caminar. } \\
\text { - (000510) Distancia de caminata. }\end{array}$ & $\begin{array}{l}\text { COMPROMISO } \\
\text { GRAVEMENTE } \\
\frac{\text { BASTANTE }}{2} \frac{\text { MEDIO }}{3} \frac{\text { POCO }}{4} \frac{\text { NADA }}{5}\end{array}$ \\
\hline 怕 & \multicolumn{3}{|c|}{$\begin{array}{l}\text { - (0221) Terapia de ejercicios: ambulación } \\
\text { - (5240) Asesoramiento. } \\
\text { - (5270) Apoyo emocional. }\end{array}$} \\
\hline \multicolumn{2}{|c|}{ ACTIVIDADES } & \multicolumn{2}{|c|}{$\begin{array}{l}\text { - El DUE animará y asesorará al paciente sobre la deambulación temprana, explicándole la necesidad de que marche } \\
\text { mayor tiempo posible y de una forma correcta. Así mismo, fomentará la participación del paciente en las AVD. } \\
\text { - El DUE permanecerá con el paciente y proporcionará sensación de seguridad durante el periodo de más ansiedad }\end{array}$} \\
\hline
\end{tabular}

\section{DOMINIO N 5: PERCEPCIÓN / COGNICIÓN.}

\begin{tabular}{|c|c|c|c|}
\hline \multicolumn{2}{|c|}{ DIAGNOSTICO } & \multicolumn{2}{|c|}{$\begin{array}{l}\text { (00126) Conocimientos deficientes } \mathbf{r} / \mathbf{c} \text { limitación cognoscitiva } \mathbf{~ m / p} \text { verbalización del problema y petición de } \\
\text { información }\end{array}$} \\
\hline & ETIQ. & $\begin{array}{l}\text { - (1814) Conocimiento: procedimientos terapéuticos. } \\
\text { - (1803) Conocimientos: Proceso de la enfermedad. }\end{array}$ & - (1813) Conocimientos: Régimen terapéutico. \\
\hline ○् & INDIC. & $\begin{array}{l}\text { - (181401) Descripción del procedimiento terapéutico. } \\
\text { - (181404) Descripción de cómo funciona el dispositivo } \\
\text { - (181407) Descripción de los cuidados adecuados del } \\
\text { equipamiento. } \\
\text { - (180311) Descripción de las prevenciones para } \\
\text { prevenir complicaciones. } \\
\text { - (180310) Descripción de signos y síntomas de las } \\
\text { complicaciones. } \\
\text { - (181305) Descripción de la dieta prescrita. } \\
\text { - (181307) Descripción de la actividad prescrita. }\end{array}$ & $\frac{\text { NINGUNO ESCASO }}{2} \frac{\text { MODERADO }}{3} \frac{\text { SUSTANCIAL }}{4} \frac{\text { EXTENSO }}{5}$ \\
\hline 빅 & $\begin{array}{l}-(5618) \\
-(5610) \\
-(1780)\end{array}$ & $\begin{array}{l}\text { nseñanza: procedimiento/ tratamiento } \\
\text { Enseñanza: prequirúrgica. } \\
\text { uidados de una prótesis. }\end{array}$ & $\begin{array}{l}\text { cilitar el aprendizaje. } \\
\text { señanza: Actividad/ejercicio prescrito. } \\
\text { eñanza: Proceso. }\end{array}$ \\
\hline
\end{tabular}




\begin{tabular}{|l|l|}
\hline & $\begin{array}{l}\text { - El DUE de la consulta de traumatología, informará al paciente sobre lo que se encontrará antes, durante y después de la } \\
\text { intervención quirúrgica, animará al paciente a que visite la unidad de encamación e incluso a que conozca al personal de dicha } \\
\text { unidad. (7) }\end{array}$ \\
- El DUE de la consulta informará al paciente sobre el utillaje domiciliario que el paciente deberá de poseer una vez operado, \\
(andador, elevador del sanitario, sillón con asiento alto, etc). \\
- Evaluar el nivel de conocimientos del paciente respecto a su intervención. \\
- Comentar los cambios en el estilo de vida para evitar complicaciones, describiendo éstas. \\
- Enseñar al paciente y ayudar en su recuperación, dándole tiempo de hacer preguntas y reforzando la información \\
proporcionada por otros miembros del equipo de cuidados. \\
- Entregar el informe de recomendaciones al alta. (Alta de enfermería. Anexo II)
\end{tabular}

\section{DOMINIO N 5 : PERCEPCIÓN / COGNICIÓN.}

\begin{tabular}{|c|c|c|c|}
\hline \multicolumn{2}{|c|}{ DIAGNOSTICO } & \multicolumn{2}{|c|}{ (00173) Riesgo de confusión aguda $\mathbf{r} / \mathbf{c}$ traumatismo o reagudización del proceso crónico. } \\
\hline & ETIQ. & $\begin{array}{l}\text { - (2102) Nivel de dolor. } \\
\text { - (0901) Orientación cognitiva. }\end{array}$ & - (0600) Equilibrio electrolítico y ácido-base. \\
\hline 气े & INDIC. & $\begin{array}{l}\text { - (090104) Identifica el día presente. } \\
\text { - (090101) Se autoidentifica. } \\
\text { - (090103) Identifica el lugar donde está. } \\
\text { - (060017) Orientación cognitiva. } \\
\text { - (060016) Alerta mental. }\end{array}$ & $\begin{array}{c}\text { COMPROMISO } \\
\frac{\text { GRAVEMENTE }}{1} \frac{\text { BASTANTE }}{2} \quad \frac{\text { MEDIO }}{3} \quad \frac{\text { POCO }}{4} \quad \frac{\text { NADA }}{5}\end{array}$ \\
\hline 它 & \multicolumn{3}{|c|}{$\begin{array}{l}\text { - (4820) Orientación de la realidad. } \\
\text { - (6440) Manejo del delirio. }\end{array}$} \\
\hline 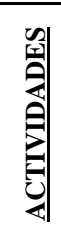 & \multicolumn{3}{|c|}{$\begin{array}{l}\text {-El DUE valorará la posible causa de la confusión o delirio con el médico responsable si fuera necesario, para tomar las } \\
\text { medidas oportunas. } \\
\text {-El personal de la planta orientará sobre la realidad, intentando razonar con el paciente si fuera posible, dando apoyo a los } \\
\text { cuidadores, promoviendo un ambiente seguro, etc. } \\
\text {-El DUE administrará la medicación pautada (analgesia, ansiolíticos, hipnóticos), dependiendo de la situación o causa. } \\
\text {-En caso necesario se tomarán medidas de tipo "sujeciones mecánicas". }\end{array}$} \\
\hline
\end{tabular}

DOMINIO N 7 : ROL / RELACIONES.

DIAGNOSTICO (00062) Riesgo de cansancio del rol cuidador, r/c la vulnerabilidad de la dificultad del rol del cuidador,

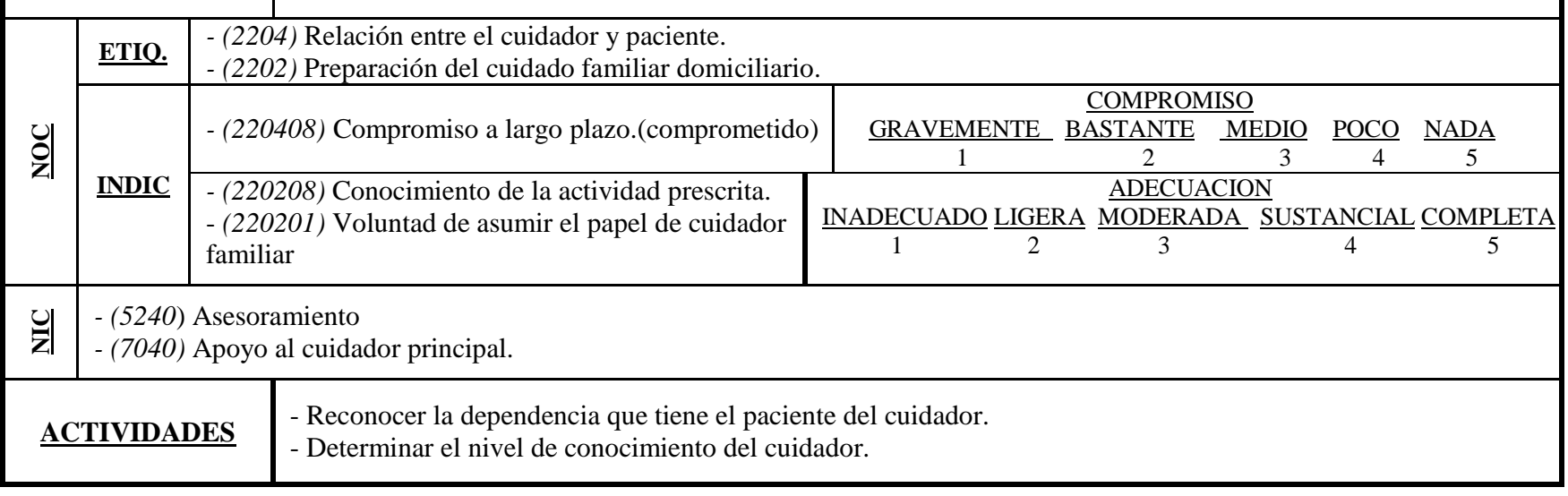

\section{DOMINIO N 9 : AFRONTAMIENTO / TOLERANCIA AL ESTRÉS.}

\begin{tabular}{|c|c|c|c|}
\hline \multicolumn{2}{|c|}{ DIAGNOSTICO } & \multicolumn{2}{|c|}{$\begin{array}{l}\text { (00074) Afrontamiento familiar comprometido r/c desorganización familiar y cambio de roles temporales } \mathbf{m} / \mathbf{p} \text { que } \\
\text { la persona de referencia describe o confirma una comprensión o conocimiento inadecuado que interfiere con las } \\
\text { conductas efectivas de ayuda y soporte o bien por expresiones de desbordamiento y preocupación familiar. }\end{array}$} \\
\hline \multirow[b]{2}{*}{ 气| } & ETIQ. & \multicolumn{2}{|c|}{$\begin{array}{l}\text { - (2200) Superación de problemas de la familia. } \\
\text { - (2204) Relación entre cuidador principal y paciente. }\end{array}$} \\
\hline & $\underline{\text { INDIC. }}$ & $\begin{array}{l}\text { - (220401) Comunicación efectiva. } \\
\text { - (220411) Solución de problemas en colaboración. } \\
\text {-(220412) Sentido de la responsabilidad. }\end{array}$ & $\frac{\text { GRAVEMENTE }}{\frac{\text { BASTANTE }}{2}} \frac{\text { MEDIO }}{3} \frac{\text { POCO }}{4} \frac{\text { NADA }}{5}$ \\
\hline$\vec{z}$ & \multicolumn{3}{|c|}{$\begin{array}{ll}\text { - (5240) Asesoramiento. } & -(7110) \text { Fomento de la implicación familiar } \\
\text { - (5250) Apoyo en toma de decisiones. } & -(7040) \text { Apoyo al cuidador principal. } \\
\text { - (5270) Apoyo emocional. } & -(7140) \text { Apoyo a la familia. }\end{array}$} \\
\hline
\end{tabular}




\begin{tabular}{|c|c|c|c|}
\hline : & \multicolumn{3}{|c|}{$\begin{array}{l}\text { - El DUE de la consulta, proporcionará información y apoyo al paciente y familiar que deben tomar una decisión, con el objetivo } \\
\text { de facilitar los cuidados primarios al paciente por parte de una persona distinta del profesional de cuidados sanitarios. } \\
\text { - El DUE de la consulta y de la unidad de hospitalización, facilitarán la participación de la familia en el cuidado emocional y } \\
\text { físico del paciente. Así mismo, comprobará que el cuidador principal tiene la aptitud y actitud necesarias para cuidar al paciente, } \\
\text { facilitando la participación de la familia en el cuidado del paciente. Se responderá a las preguntas del paciente y cuidador. }\end{array}$} \\
\hline \multicolumn{2}{|c|}{$\underline{\text { DIAGNOSTICO }}$} & \multicolumn{2}{|c|}{$\begin{array}{l}\text { (00146) Ansiedad } \mathbf{r} / \mathbf{c} \text { cambio en el estado de salud } \mathbf{m} / \mathbf{p} \text { dificultad para conciliar el sueño y miedo de } \\
\text { consecuencias inespecíficas y nerviosismo. }\end{array}$} \\
\hline \multirow{4}{*}{ 乌్ } & ETIQ. & $\begin{array}{l}\text { - (1402) Autocontrol de la ansiedad (a nivel prec } \\
\text { - (1211) Nivel de ansiedad. }\end{array}$ & erotorio (P) y postoperatoriol \\
\hline & \multirow{3}{*}{$\underline{\text { INDIC. }}$} & $\begin{array}{l}\text { - (121117) Ansiedad verbalizada. } \\
\text { - (121105) Inquietud. }\end{array}$ & $\frac{\text { GRAVE }}{1} \frac{\text { SUSTANCIAL }}{2} \frac{\text { MODERADO }}{3} \frac{\text { LEVE }}{4} \frac{\text { NINGUNO }}{5}$ \\
\hline & & $\begin{array}{l}\text { - (140214) Refiere dormir de forma adecuada. } \\
\text { - (140217) Controla la respuesta de ansiedad. } \\
\text { - (140204) Busca información para reducir la } \\
\text { ansiedad. }\end{array}$ & $\begin{array}{l}\text { DEMOSTRACIÓN. } \\
\text { NUNCA RARAMENTE A VECES FRECUENTE SIEMPRE }\end{array}$ \\
\hline & & $\begin{array}{l}\text { - (140216) Ausencia de manifestaciones de una } \\
\text { conducta de ansiedad. }\end{array}$ & 2 \\
\hline 至 & \multicolumn{3}{|c|}{$\begin{array}{l}\text { - (5820) Disminución de la ansiedad. } \\
\text { - (2340) Administración de medicación. Oral. } \\
\text { - (5240) Asesoramiento. } \\
\text { - (5618) Enseñanza: Procedimiento/tratamiento. }\end{array}$} \\
\hline 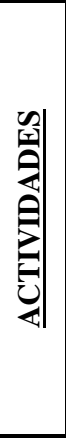 & \multicolumn{3}{|c|}{$\begin{array}{l}\text { - El DUE advertirá al paciente que tras la intervención, pasará a la sala de reanimación donde lo monitorizarán y estará rodeado de } \\
\text { aparatos y sonidos extraños. } \\
\text { - El DUE advertirá al paciente de la necesidad de permanecer en dieta absoluta desde las } 24 \text { horas del día precedente a la } \\
\text { intervención. El AE podrá el cartel de ayunas a los pies de la cama la tarde anterior a la intervención. } \\
\text { - El DUE informará al paciente de la hora a la que se le preparará para la intervención, así como en qué constará dicha } \\
\text { preparación ( aseo personal, cambio de lencería de la cama, colocación de sonda vesical, instauración de enema tipo cassen, } \\
\text { colocación de sueroterapia preescrita y medicación preoperatoria y retirada de objetos personales y prótesis dentales). } \\
\text { - El DUE ofrecerá al paciente la benzodiazepina prescrita por el anestesista a las } 23 \text { horas del día precedente a la intervención y } \\
\text { en días posteriores a la misma hora si precisa. Tranquilizará al paciente sobre mitos relativos a los analgésicos (dependencia, } \\
\text { depresión respiratoria, etc.) } \\
\text { - El DUE informará al paciente sobre su posterior recuperación y disipará los temores del paciente respecto a la luxación de } \\
\text { cadera, asegurándole que con unas correctas medidas ergonómicas, esa complicación no tiene porqué ocurrir. }\end{array}$} \\
\hline
\end{tabular}

\section{DOMINIO N 10: PRINCIPIOS VITALES.}

\begin{tabular}{|c|c|c|c|}
\hline \multicolumn{2}{|c|}{ DIAGNOSTICO } & \multirow{2}{*}{\multicolumn{2}{|c|}{$\begin{array}{l}\text { (00083) Conflicto de decisiones } \mathbf{r} / \mathbf{c} \text { percepción de amenaza a valores personales } \mathbf{~ m / p} \text { vacilación entre } \\
\text { expresiones alternativas } \\
\text { - (0906) Toma de decisiones. }\end{array}$}} \\
\hline \multirow[b]{2}{*}{ z) } & ETIQ. & & \\
\hline & INDIC. & $\begin{array}{l}\text { - (090601) Identifica información relevante. } \\
\text { - (090602) Identifica alternativas. } \\
\text { - (090604) Identifica información relevante. }\end{array}$ & $\frac{\text { GOMPROMISO }}{\text { GRAVEMENTE }} \frac{\text { BASTANTE }}{2} \frac{\text { MEDIO }}{3} \frac{\text { POCO }}{4} \frac{\text { NADA }}{5}$ \\
\hline$\underline{\text { NIC }}$ & \multicolumn{3}{|c|}{ - (5250) Apoyo en toma de decisiones. } \\
\hline 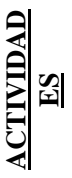 & \multicolumn{3}{|c|}{$\begin{array}{l}\text { - El DUE de la consulta, proporcionará información y apoyo al paciente y familiar que deben tomar una decisión, con el objetivo } \\
\text { de facilitar los cuidados primarios al paciente por parte de una persona distinta del profesional de cuidados sanitarios. } \\
\text { - El DUE de la consulta y de la unidad de hospitalización, facilitarán la participación de la familia en el cuidado emocional y } \\
\text { físico del paciente. } \\
\text { - El DUE comprobará que el cuidador principal tiene la aptitud y actitud necesarias para cuidar al paciente }\end{array}$} \\
\hline
\end{tabular}

DOMINIO No 11 : SEGURIDAD / PROTECCIÓN.

\begin{tabular}{|c|c|c|c|c|c|c|c|}
\hline \multicolumn{2}{|c|}{ DIAGNOSTICO } & \multicolumn{6}{|c|}{ (00086) Riesgo de disfunción neurovascular periférica r/c cirugía ortopédica } \\
\hline \multirow[b]{2}{*}{ Z्. } & ETIQ. & - (0407) Perfusión tisular: Periférica & & & & & \\
\hline & INDIC. & $\begin{array}{l}\text { - (040716) Llenado capilar de los dedos de los pies. } \\
\text { - (040710) Temperatura de extremidades caliente. } \\
\text { - (040706) Sensibilidad. }\end{array}$ & $\frac{\text { GRAVEMENT }}{1}$ & $\frac{\begin{array}{r}\text { COMPR } \\
\text { BASTANTE }\end{array}}{2}$ & $\frac{\text { MISO }}{\frac{\text { MEDIO }}{3}}$ & $\frac{\mathrm{POCO}}{4}$ & $\frac{\mathrm{NADA}}{5}$ \\
\hline$\underline{\text { NIC }}$ & \multicolumn{7}{|c|}{ - (2660) Gestión de sensibilidad periférica. } \\
\hline \multicolumn{2}{|c|}{ ACTIVIDADES } & \multicolumn{6}{|c|}{$\begin{array}{l}\text { - El DUE vigilará la sensibilidad y color de la piel del miembro operado así como la movilidad de dedos y tobillo a } \\
\text { la llegada a planta y posteriormente cada } 8 \text { horas. }\end{array}$} \\
\hline
\end{tabular}




\begin{tabular}{|c|c|c|c|}
\hline \multicolumn{2}{|c|}{ DIAGNOSTICO } & \multicolumn{2}{|c|}{$\begin{array}{l}\text { (00005) Riesgo de desequilibrio de temperatura corporal r/c enfermedad o traumatismo que afecta a la regulación } \\
\text { de la temperatura, sedación e inactividad }\end{array}$} \\
\hline \multirow[b]{2}{*}{ ○्ञ } & ETIQ. & \multicolumn{2}{|l|}{ - (0800) Termorregulación } \\
\hline & $\underline{\text { INDIC. }}$ & $\begin{array}{l}\text { - (080001) Temperatura cutánea aumentada. } \\
\text { - (080014) Deshidratación. } \\
\text { - (080019) Hipertermia. } \\
\text { - (080020) Hipotermia. }\end{array}$ & $\frac{\text { GRAVE }}{1} \frac{\text { SUSTANCIAL }}{2} \frac{\text { MODERADO }}{3} \frac{\text { LEVE }}{4} \frac{\text { NINGUNO }}{5}$ \\
\hline$\underline{\mathrm{NIC}}$ & \multicolumn{3}{|c|}{$\begin{array}{l}\text { - (3740) Tratamiento de la fiebre. } \\
\text { - (3990) Regulación de la temperatura. } \\
\text { - (6680) Monitorización de signos vitales }\end{array}$} \\
\hline \multicolumn{2}{|c|}{$\underline{\text { ACTIVIDADES }}$} & \multicolumn{2}{|c|}{$\begin{array}{l}\text { - El AE tomará y registrará la temperatura del paciente a su llegada a planta y posteriormente cada } 8 \text { horas. } \\
\text { - El personal del servicio mantendrá una temperatura adecuada en la habitación. } \\
\text { - El DUE administrará antitérmico prescrito y medios físicos en caso necesario. }\end{array}$} \\
\hline \multicolumn{2}{|c|}{ DIAGNOSTICO } & \multicolumn{2}{|c|}{$\begin{array}{l}\text { (00047) Riesgo de deterioro de la integridad cutánea } \mathbf{r} / \mathbf{c} \text { herida quirúrgica, presencia de drenajes e inmovilidad } \\
\text { física. }\end{array}$} \\
\hline \multirow{3}{*}{ 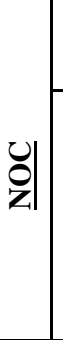 } & ETIQ. & $\begin{array}{l}\text { 02) Curación de la herida por } 1^{a} \text { intención. } \\
\text { 01) Integridad tisular: piel y mucosas }\end{array}$ & \\
\hline & \multirow[t]{2}{*}{$\underline{\text { INDIC. }}$} & $\begin{array}{l}\text { - (110213) Aproximación de bordes de heridas } \\
\text { - (110205) Secreción serosanguinolenta. } \\
\text { - (110209) Edema perilesional. } \\
\text { - (110208) Eritema cutáneo circundante. }\end{array}$ & $\begin{array}{llll}\text { NINGUNO } & \text { ESCASO } \\
2 & \frac{\text { MODERADO }}{3} & \frac{\text { SUSTANCIAL }}{4} & \text { EXTENSO } \\
5\end{array}$ \\
\hline & & $-(110121)$ Eritema & 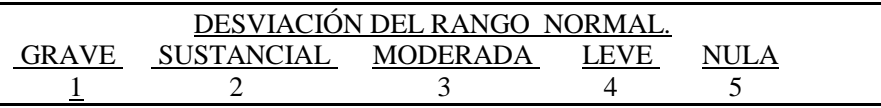 \\
\hline 블 & \multicolumn{3}{|c|}{$\begin{array}{ll}\text { - (3440) Cuidados del sitio de incisión. } & -(3584) \text { Cuidados de la piel: Tratamiento tópico. } \\
\text { - (3660) Cuidados de las heridas. } & -(3540) \text { Prevención de úlceras por presión. } \\
\text { - (3662) Cuidado de las heridas con drenaje cerrado. } & -(1870) \text { Cuidados del drenaje } \\
\end{array}$} \\
\hline 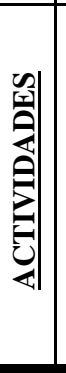 & \multicolumn{3}{|c|}{$\begin{array}{l}\text {-El DUE inspeccionará el sitio de incisión por si hubiera enrojecimiento, inflamación, signos de dehiscencia o sangrado. } \\
\text {-El DUE, cada } 24 \text { hs., limpiará la zona de incisión con solución antiséptica apropiada, desde la zona más limpia a la menos limpia y } \\
\text { aplicará un apósito apropiado para cubrir el sitio de incisión. } \\
\text { - -El DUE, limpiará la zona que rodea cualquier tipo de drenaje y mantendrá la posición de dicho tubo para que sea permeable (en } \\
\text { caso de tenerlo) y el AE, anotará las características y el volumen del drenaje cada } 24 \text { horas (a las } 19 \text { horas). } \\
\text {-El AE, mantendrá la ropa de la cama limpia, seca y sin arrugas. Eliminará la humedad excesiva causada por transpiración, drenaje } \\
\text { de heridas e incontinencia fecal y urinaria. Utilizará jabón de Ph adecuado a la piel y aplicará masaje con crema hidratante. } \\
\text {-El DUE registrará estado de la piel al ingreso y después a diario, aplicando el protocolo de úlceras por presión y protecciones de } \\
\text { talones en caso necesario. } \\
\text {-El DUE retirará el drenaje a las } 48 \text { horas de la intervención, por prescripción facultativa. }\end{array}$} \\
\hline \multicolumn{2}{|c|}{ DIAGNOSTICO } & \multicolumn{2}{|c|}{ (00004) Riesgo de infección $\mathbf{r} / \mathbf{c}$ procedimientos invasivos y traumatismo. } \\
\hline \multirow{3}{*}{ : } & ETIQ. & \multicolumn{2}{|l|}{$\begin{array}{l}\text { - (0702) Estado inmune. } \\
\text { - (1807) Conocimiento: control de la infección. }\end{array}$} \\
\hline & \multirow[b]{2}{*}{$\underline{\text { INDIC }}$} & $\begin{array}{l}\text { - (070207) Temperatura corporal . } \\
\text { - (070208) Integridad cutánea. }\end{array}$ & $\begin{array}{lllll}\frac{\text { COMPROMISO }}{\text { GRAVEMENTE }} & \frac{\text { BASTANTE }}{2} & \frac{\text { MEDIO }}{3} & \frac{\text { POCO }}{4} & \frac{\text { NADA }}{5}\end{array}$ \\
\hline & & $\begin{array}{l}\text { - (180703) Descripción de las practicas que } \\
\text { reducen la transmisión } \\
\text { - (180707) Descripción de las actividades para } \\
\text { aumentar la resistencia a la infección }\end{array}$ & $\frac{\text { NINGUNO }}{1} \frac{\text { ESCASO }}{2} \frac{\text { MODERADO }}{3} \frac{\text { SUSTANCIAL }}{4} \frac{\text { EXTENSO }}{5}$ \\
\hline 穵 & \multicolumn{3}{|c|}{$\begin{array}{l}\text { - (6520).-Análisis de la situación sanitaria. } \\
\text { - (6650).-Vigilancia. } \\
\text { - (5510).-Educación sanitaria. } \\
\text { - (6480).-Manejo ambiental. }\end{array}$} \\
\hline 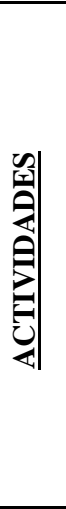 & \multicolumn{3}{|c|}{$\begin{array}{l}\text {-El DUE revisará la historia clínica y recopilará datos del paciente para detectar problemas de salud que aumenten el riesgo de } \\
\text { infección. Se vigilará el proceso de curación del sitio de incisión, inspeccionando la existencia de enrojecimiento, calor y edema, } \\
\text { vigilando los signos y síntomas de infección (control de temperatura, etc.) a intervalos regulares. En el } 3^{\circ} \text { día, se curará la herida } \\
\text { quirúrgica y posteriormente cada } 24 \text { horas o cada vez que se estime oportuno si hay abundante exudado. } \\
\text {-El personal sanitario adoptará medidas universales de higiene para evitar contagios. } \\
\text {-El DUE realizará todas las técnicas de forma aséptica, utilizando material estéril, siguiendo los protocolos del Hospital. } \\
\text {-Se instruirá al paciente y familiares acerca de las técnicas correctas del lavado de manos e higiene en general. } \\
\text {-El DUE instruirá al paciente y familia sobre los signos y síntomas de la infección y cuando debe informar al DUE en caso de } \\
\text { que aparezcan. } \\
\text {-Limitar el número de visitas si es necesario. } \\
\text {-El DUE garantizará una manipulación aséptica de todas las vías, catéteres, drenajes y sondas. } \\
\text {-El DUE cambiará los equipos de fluidoterapia c/24h. (sistema de suero, llaves, etc.), así como vigilará el punto de punción de la } \\
\text { vía venosa, según protocolo de AVP del CHUAB. } \\
\text {-El DUE retirará sonda vesical el } 2^{\circ} \text { día postintervención. }\end{array}$} \\
\hline
\end{tabular}


DOMINIO No 11 : SEGURIDAD / PROTECCIÓN. (bis)

\begin{tabular}{|c|c|c|c|}
\hline \multicolumn{2}{|c|}{$\underline{\text { DIAGNOSTICO }}$} & \multicolumn{2}{|c|}{$\begin{array}{l}\text { (00155) Riesgo de caída } \mathbf{r} / \mathbf{c} \text { prótesis en miembros inferiores, uso de dispositivos de ayuda o deterioro de la } \\
\text { movilidad física }\end{array}$} \\
\hline \multirow{4}{*}{ 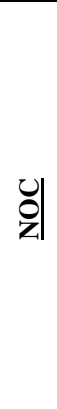 } & ETIQ. & $\begin{array}{l}\text { - (1911) Conducta de seguridad: personal. } \\
\text { - (1909) Conducta de seguridad: prevención } \\
\text { - (1809) Conocimientos: seguridad personal }\end{array}$ & $\begin{array}{l}\text { - (1912) Estado de seguridad: caídas. } \\
\text { - (0208) Nivel de movilidad }\end{array}$ \\
\hline & \multirow{3}{*}{$\underline{\text { INDIC. }}$} & $\begin{array}{l}\text { - (191107) Uso correcto de dispositivos de } \\
\text { ayuda. }\end{array}$ & $\begin{array}{l}\text { DEMOSTRACIÓN. } \\
\frac{\text { NUNCA }}{1} \frac{\text { RARAMENTE }}{2} \frac{\text { A VECES }}{3} \frac{\text { FRECUENTE }}{4} \frac{\text { SIEMPRE }}{5}\end{array}$ \\
\hline & & $\begin{array}{l}\text { - (020801) Mantenimiento del equilibrio. } \\
\text { - (020809) Coordinación. }\end{array}$ & $\begin{array}{c}\text { COMPROMISO } \\
\frac{\text { GRAVEMENTE }}{1} \frac{\text { BASTANTE }}{2}\end{array} \frac{\text { MEDIO }}{3} \quad \frac{\text { POCO }}{4} \quad \frac{\text { NADA }}{5}$ \\
\hline & & $\begin{array}{l}\text { - (180902) Descripción de medidas de } \\
\text { prevención de caídas }\end{array}$ & $\frac{\text { NINGUNO ESCASO }}{1} \frac{\text { MODERADO }}{3} \frac{\text { SUSTANCIAL }}{4} \frac{\text { EXTENSO }}{5}$ \\
\hline 気 & \multicolumn{3}{|c|}{$\begin{array}{l}\text { - (6610) Identificación de riesgos. } \\
\text { - (5606) Enseñanza: individual. } \\
\text { - (6490) Prevención de caídas. }\end{array}$} \\
\hline 窇 & \multicolumn{3}{|c|}{$\begin{array}{l}\text { - El DUE ayudará al paciente a desarrollar un programa de ejercicios de acuerdo a su edad y estado físico, y también animará } \\
\text { a mantener una base de apoyo amplia. } \\
\text { - El personal de la unidad facilitará un ambiente seguro para la práctica de ejercicios y vigilará la respuesta del paciente a los } \\
\text { mismos. } \\
\text { - El DUE planificará las actividades de eliminación de riesgos en colaboración con el paciente. } \\
\text { - El DUE vigilará la marcha, equilibrio y cansancio en la deambulación del paciente. } \\
\text { - El DUE valorará las capacidades e incapacidades psicomotoras y ayudará (salvo contraindicación) en la bipedestación / } \\
\text { sedestación y en el balanceo lateral del cuerpo para estimular el equilibrio corporal. }\end{array}$} \\
\hline
\end{tabular}

DOMINIO N ${ }^{\circ}$ 12: CONFORT.

\begin{tabular}{|c|c|c|c|}
\hline \multicolumn{2}{|c|}{$\underline{\text { DIAGNOSTICO }}$} & \multicolumn{2}{|c|}{ (00183) Disposición para mejorar el confort $\mathbf{r} / \mathbf{c}$ cambio en el entorno $\mathbf{m} / \mathbf{p}$ nerviosismo. } \\
\hline \multirow[b]{2}{*}{ そ) } & ETIQ. & \multicolumn{2}{|c|}{$\begin{array}{l}\text { - (3000) Satisfacción del paciente/usuario: acceso a los recursos asistenciales. } \\
\text { - (3001) Satisfacción del paciente/usuario: cuidados }\end{array}$} \\
\hline & INDIC. & $\begin{array}{l}\text { - (300006) Acceso a la enfermera responsable de los cuidados. } \\
\text { - (300005) Informado de la enfermera y del personal auxiliar } \\
\text { responsable. } \\
\text { - (300001) Disponibilidad de las enfermeras. } \\
\text { - (300114) Orientación en la habitación, equipo y rutinas. } \\
\text { - (300108) Relación con el personal de enfermería. }\end{array}$ & $\begin{array}{ll}\text { NADA } & \frac{\text { GRADO DE SATISFACCIÓN. }}{1} \frac{\text { REGULAR }}{2} \frac{\text { BASTANTE }}{3} \frac{\text { MUCHO }}{5}\end{array}$ \\
\hline 비 & \multicolumn{3}{|c|}{$\begin{array}{l}\text { - (6482) Manejo ambiental: confort } \\
\text { - (7310) Cuidados de enfermería al ingreso. }\end{array}$} \\
\hline 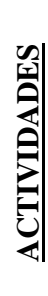 & \multicolumn{3}{|c|}{$\begin{array}{l}\text { - El auxiliar de enfermería (AE) acompañará al paciente a su habitación y lo proveerá de los útiles de aseo personal. Colocará } \\
\text { pulsara identificativa al paciente en el lado de la intervención quirúrgica. } \\
\text { - El DUE le enseñará al paciente el funcionamiento de las instalaciones de la habitación (timbre y pulsador de luz, mando de la } \\
\text { cama, etc...) } \\
\text { - El DUE resolverá las dudas del paciente referentes a la intervención y le asegurará la completa disposición de todo el personal } \\
\text { para con su cuidado. } \\
\text { - El DUE y AE aplicará el Plan de Acogida vigente al paciente y familiares. } \\
\text { - El DUE realizará la Valoración del paciente (Anexo I) y la toma de constantes vitales. }\end{array}$} \\
\hline
\end{tabular}

DOMINIO No 12 : CONFORT.

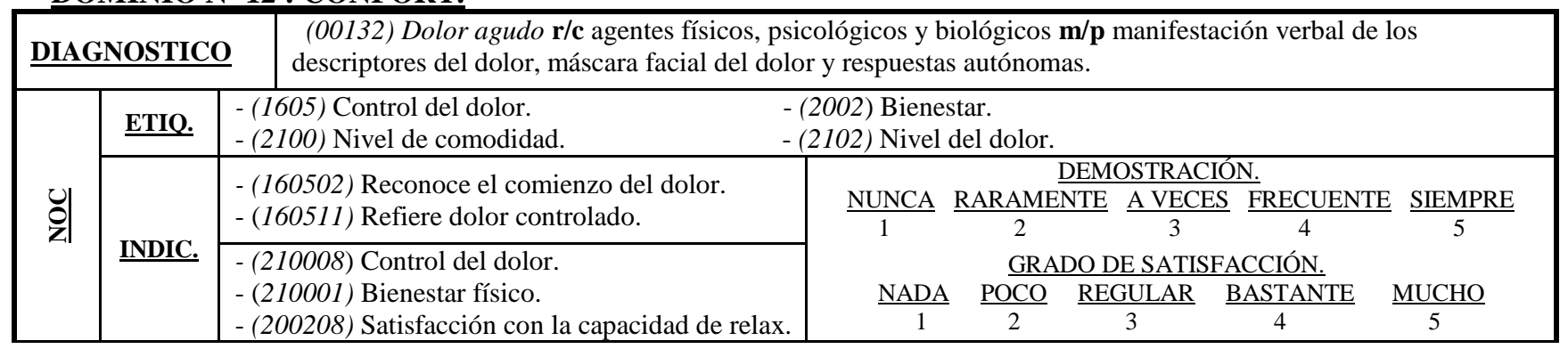




\begin{tabular}{|c|c|c|c|}
\hline 빔 & \multicolumn{3}{|c|}{$\begin{array}{l}\text { - (1400) Manejo del dolor. } \\
\text { - (2380) Manejo de la medicación. } \\
\text { - (2210) Administración de analgésicos. } \\
\text { - (2400) Asistencia en la analgesia. controlada por el paciente (ACP). }\end{array}$} \\
\hline 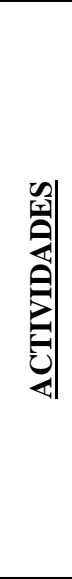 & \multicolumn{3}{|c|}{$\begin{array}{l}\text { - El DUE valorará el dolor del paciente a su llegada a planta y durante su permanencia en ésta, incluyendo la localización, } \\
\text { características, duración, frecuencia, expresiones no verbales de dolor, y evaluará las medidas antiálgicas. Informará al } \\
\text { paciente y familiares de las posturas antiálgicas (decúbito supino y miembro operado en abducción y rotación externa). } \\
\text { - El DUE informará al paciente sobre la conveniencia de avisar al DUE en el momento de que empiece a notar dolor para la } \\
\text { inmediata administración de analgésico de rescate y evitar así el aumento del dolor y su consiguiente aumento de dosis } \\
\text { analgésica. } \\
\text { - El DUE preparará, administrará, y evaluará los analgésicos preescritos (tanto los de vía intravenosa como subcutánea), de } \\
\text { forma segura y efectiva, pautándolos de forma alterna los pertenecientes a distinto grupo químico. Al 3o día, se administrará el } \\
\text { analgésico vía oral. } \\
\text { - El DUE monitorizará y registrará el grado de satisfacción del paciente respecto al dolor. } \\
\text { - El personal de la unidad proporcionará un ambiente tranquilo y relajado al paciente, desaconsejando visitas familiares } \\
\text { excesivas y disminuyendo el ruido en la habitación. } \\
\text { - El DUE explicará al paciente a su llegada a planta, el funcionamiento de la bomba de ACP y de la posibilidad de } \\
\text { suministrarse él mismo de forma segura las dosis de rescate (si el paciente es portador). El infusor intravenoso se retirará a las } \\
24 \text { horas postintervención y el infusor intratecal se retirará dejando un margen de } 6 \text { horas antes y } 12 \text { horas después de la } \\
\text { administración de dosis de HBPM. (15) }\end{array}$} \\
\hline \multicolumn{2}{|c|}{ DIAGNOSTICO } & \multicolumn{2}{|c|}{$\begin{array}{l}\text { (00134) Nauseas } \mathbf{r} / \mathbf{c} \text { anestesia postquirúrgica, hipotensión ortostática, dolor y movilización } \mathbf{m} / \mathbf{p} \\
\text { sintomatología vegetativa e informes de "nauseas". }\end{array}$} \\
\hline \multirow{3}{*}{ ? } & ETIQ. & & \\
\hline & \multirow[b]{2}{*}{ INDIC. } & $\begin{array}{l}\text { - (161801) Reconoce inicio de nauseas. } \\
\text { - (161812) Informa de nauseas, esfuerzos para } \\
\text { vomitar }\end{array}$ & $\begin{array}{l}\text { DEMOSTRACIÓN. } \\
\frac{\text { IUNCA }}{1} \frac{\text { RARAMENTE }}{2} \frac{\text { A VECES }}{3} \frac{\text { FRECUENTE }}{4} \frac{\text { SIEMPRE }}{5}\end{array}$ \\
\hline & & $\begin{array}{l}\text { - (100411) Hidratación. } \\
\text { - (100401) Ingestión de nutrientes. }\end{array}$ & 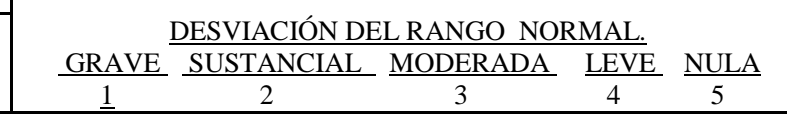 \\
\hline 블 & \multicolumn{3}{|c|}{$\begin{array}{l}\text { - (2380) Manejo de la medicación. } \\
\text { - (1450) Manejo de las nauseas. } \\
\text { - (1570) Manejo del vómito. } \\
\end{array}$} \\
\hline |ר: & \multicolumn{3}{|c|}{$\begin{array}{l}\text { - El DUE registrará y valorará la frecuencia y características de las nauseas y vómitos. } \\
\text { - El DUE administrará el antiemético prescrito. } \\
\text { - El DUE colocará al paciente de forma adecuada para prevenir la aspiración del vómito, manteniendo vías aéreas permeables. } \\
\text { - El AE limpiará al paciente, en caso necesario, evitando movimientos bruscos para no favorecer el vómito. } \\
\text { - El paciente deberá quedarse incorporado en la cama alrededor de } 1 \text { hora previa sedestación, lo cual se hará antes de comer y } \\
\text { evitando movimientos bruscos. }\end{array}$} \\
\hline
\end{tabular}




\begin{tabular}{|c|c|}
\hline \multicolumn{2}{|c|}{ REQUERIMIENTOS TERAPEUTICOS } \\
\hline$\underline{\mathrm{NIC}}$ & ACTIVIDADES. ( por orden cronológico) \\
\hline $\begin{array}{l}\text { - (2315) Administración de medicación: } \\
\text { rectal. } \\
\text { - (2304) Administración de medicación: } \\
\text { oral. } \\
\text { - (4200) Terapia intravenosa. } \\
\text { - (0580) Sondaje vesical. } \\
\text { - (2930) Preparación quirúrgica. } \\
\text { - (4190) Punción intravenosa }\end{array}$ & $\begin{array}{l}\text { - El DUE cumplimentará la hoja de enfermería de preparación prequirúrgica la tarde } \\
\text { previa a la intervención, y comprobará que el paciente tiene el preoperatorio } \\
\text { correctamente cumplimentado así como las autorizaciones de intervención quirúrgica y de } \\
\text { anestesia. } \\
\text { - El DUE, la tarde anterior a la intervención, instaurará vía venosa en brazo contralateral } \\
\text { al miembro inferior a operar según el protocolo de dispositivos de acceso venoso (DAV) } \\
\text { del CHUAB. } \\
\text { - El AE administrará al paciente el enema de limpieza la tarde anterior a la intervención } \\
\text { (18 horas), nunca la misma mañana de la intervención. } \\
\text { - El DUE tomará las constantes vitales del paciente (tensión arterial, frecuencia cardiaca } \\
\text { y temperatura) la misma mañana de la intervención ( } 7 \text { horas) anotándolas en la hoja de } \\
\text { preparación prequirúrgica. } \\
\text { - El DUE administrará la medicación oral preescrita al paciente la misma mañana de la } \\
\text { intervención (a las } 7 \text { horas) debiendo de ser ingerida con la mínima agua posible. } \\
\text { - El DUE administrará la medicación intravenosa preescrita por anestesia y dejará } \\
\text { preparada (sin comenzar perfusión) la profilaxis antibiótica preescrita por el } \\
\text { traumatólogo, siendo ésta: (9) (10) } \\
\text { - Cefazolina } 2 \text { gramos IV. ( empezar a infundir a la entrada en quirófano) } \\
\text { - Vancomicina } 1 \text { gramo IV en caso de pacientes alérgicos a penicilinas y en } \\
\text { pacientes portadores de Staphiloccocus aureus meticilín resistente. (empezar a } \\
\text { infundir } 1 \text { hora antes de intervención.) } \\
\text { - El DUE colocará sonda vesical la misma mañana de la intervención según el protocolo } \\
\text { en vigor en el CHUAB. }\end{array}$ \\
\hline
\end{tabular}

\begin{tabular}{|c|c|}
\hline \multicolumn{2}{|c|}{ REQUERIMIENTOS DIAGNÓSTICOS. } \\
\hline \multicolumn{1}{|c|}{ NIC } & ACTIVIDADES. \\
\hline - (4238) Flebotomía. & $\begin{array}{l}\text { - El DUE, la tarde previa a la intervención, extraerá sangre para realizar pruebas } \\
\text { cruzadas en caso necesario y analítica preoperatoria en caso de que no estuviera hecha. }\end{array}$ \\
\hline
\end{tabular}

\begin{tabular}{|l|l|}
\hline \multicolumn{2}{|c|}{ COMPLICACIONES POTENCIALES Y DIAGNÓSTICOS A LAS QUE SE REFIEREN } \\
\hline \multicolumn{1}{|c|}{ DIAGNÓSTICOS NANDA. } & \multicolumn{1}{|c|}{ COMPLICACIONES POTENCIALES. } \\
\hline - (00085) Deterioro de la movilidad física r/c disminución de \\
$\begin{array}{l}\text { la fuerza, dolor, miedo a iniciar el movimiento m/p dificultad } \\
\text { para realizar las actividades del autocuidado. }\end{array}$ \\
$\begin{array}{l}\text { (1002201) Hemorragia / formación de hematomas. } \\
\text { - (10002203) Dislocación / subluxación articular. } \\
\text { herida quirún neurovascular. }\end{array}$ \\
\hline
\end{tabular}

\section{CONCLUSIONES}

Tras la consulta de la bibliografía reseñada y la experiencia profesional del equipo de trabajo, hemos concluido que el presente Plan de Cuidados Estándar de Enfermería para artroplastia de cadera, cumple con los objetivos expuestos en un apartado anterior (ver Objetivos), pudiendo ser una herramienta muy valiosa en la planificación de la atención al paciente, en el desarrollo de dicha atención y en una posterior evaluación de los objetivos propuestos. La consecución de dichos objetivos, a juicio de los miembros del equipo, implicaría una mejor y más rápida recuperación del paciente. 


\section{BIBLIOGRAFÍA}

1.- Osakidetza, Fundación Signo. Manual de descripción de los grupos relacionados por el diagnóstico (AP-GRD) [programa de ordenador]. Versión 21.0. Administración de la Comunidad Autónoma del País Vasco; 2007.

2.- Ten Gil A, Campos Adrian E, Casañ Benito A, Gonzalez Sanchez E. Cuidados de enfermería en pacientes intervenidos de artroplastia de cadera en el CHGUV. Enfermería integral [en línea] 2007 Diciembre [fecha de acceso 19 de febrero 2011]; (80): 37-40.

Disponible en: http://www.enfervalencia.org/ei/80/articulos-cientificos/10.pdf

3.- NANDA International, Diagnósticos enfermeros: Definición y Clasificación, 2009-2011. Madrid: Elservier; 2010.

4.- McCloskey Joanne C, Bulechek G.. Clasificación de intervenciones de enfermería (NIC). 4a edición. Madrid: Elservier; 2010.

5.- Johnson M, Bulechek G, Butcher H, Dochterman JM. Interrelaciones NANDA, NOC y NIC: diagnósticos enfermeros, resultados e intervenciones. $2^{a}$ edición. Madrid: Elservier; 2007.

6 .- Gordon M. Manual de diagnósticos de enfermería. 11ª edición. Madrid: Mcgraw-Hill / Interamericana De España, S.A.; 2007.

7.- Bailey L. Actualización en cuidados traumatológicos. Nursing.2004;22 (1): 14-19.

Trebon S, Medina Porqueres I, Jurado Bueno A. Enfoque multidisciplinar en las endoprótesis de cadera y rodilla: un nuevo concepto. Fisioterapia. 2001; 23(3) 113-120.

8 .- McDonald S, Green SE, Hetrick S. Pre-operative education for hip or knee replacement. Cochrane Database Syst Rev. 2004

9.- Jämsen $\mathrm{E}$, et all. Prevention of deep infection in joint replacement surgery. A review. Acta Orthop. 2010 Dec;81:660-6. Review.

10.- Meehan J, Jamali AA, Nguyen H. Prophylactic antibiotics in hip and knee arthroplasty. J Bone Joint Surg Am. 2009 Oct;91:2480-90. Review.

11.- Fdez-Lasquetty B. Recomendaciones tras la cirugía de cadera. Enfertrauma.es [en línea] 2006 [fecha de acceso 18 de febrero de 2011]. URL disponible en:

http://enfertrauma.es/pag/05 recomenCadera.html

12.- Rogers BA, Little NJ. Thromboprophylaxis in orthopedia surgery: a clinical review. J Perioper Pract. 2010 Oct;20:358-62

13 .- Hitos K, Fletcher J.P. Venous thromboembolism following primary total hip arthroplasty. Int Angiol. 2009 Jun;28:215-21.

14 .- Monte Secades, Rafael; Rabuñal Rey, Ramón; Bal Alvaredo, Mercedes y Guerrero Lombardía, José: Guía clínica para la atención de los pacientes con fractura de cadera. [en línea] [fecha de acceso 12 abril 2011]. Disponible en

www.meiga.info/guias/FracturaCadera.asp

15 .- MA Gómez Ríos, L Nieto. Analgesia epidural frente a analgesia endovenosa en cirugía ginecológica oncológica. Rev electrónica AnestesiaR 2010; Vol 2(8): 84 


\section{ANEXO I}

\section{VALORACIÓN DE ENFERMERÍA PATRONES FUNCIONALES DE MARJORIE GORDON}

APELLIDOS:

NOMBRE:

FECHA:

NHC:

EDAD:

DIAGNÓSTICO MÉDICO (DSM-IV):

ALTERACIONES SOMÁTICAS:

TRATAMIENTO FARMACOLÓGICO:

TERAPEUTA REFERENTE:

ENFERMERA REFERENTE:

CONSTANTES: TA: FC:

\section{PATRÓN DE PERCEPCIÓN-CONTROL DE LA SALUD:}

ASPECTO GENERAL: Adecuado $\square$ Descuidado $\square$ Extravagante $\square$ Meticuloso HIGIENE PERSONAL: Adecuada $\square$ Deficiente $\square$

HÁBITOS HIGIÉNICOS:

Ducha $\square$ Frecuencia __ Higiene dental $\square$ Frecuencia

Baño $\square$ Frecuencia _ Higiene cabello $\square$ Frecuencia

ACTITUD ANTE LA ENFERMEDAD: Aceptación $\square$ Rechazo $\square$ Indiferencia $\square$

Sobrevaloración $\square$ Negación $\square$

ADAPTACIÓN AL RÉGIMEN TERAPÉUTICO:

Cumple visitas: $\mathrm{Si} \square$ No $\square$

Cumple tratamiento farmacológico: $\mathrm{Si} \square$ No $\square$

Causas del incumplimiento:

ADAPTACIÓN PREVIA A RÉGIMEN TERAPÉUTICO:

Cumplía visitas: $\mathrm{Si} \square$ No $\square$

Cumplía tratamiento farmacológico: $\mathrm{Si} \square \mathrm{No} \square$

Causas del incumplimiento:

CONSUMO DE TÓXICOS:

Alcohol $\square$ Cantidad / día

Tabaco $\square$ Cantidad / día Otros

DATOS DE INTERÉS:

Otros
Cantidad / día Cantidad / día

\section{PATRÓN NUTRICIONAL-METABÓLICO:}

PESO: TALLA:

HÁBITOS ALIMENTARIOS:

DESAYUNO:

ALMUERZO:

COMIDA:

MERIENDA:

CENA:

VALORACIÓN TIPO DE DIETA: Adecuada $\square$ Inadecuada $\square$

INGESTA: Normal $\square$ Aumentada $\square$ Disminuida $\square$ Autónoma $\square$ Asistida $\square$

DATOS DE INTERÉS:

\section{PATRÓN DE ELIMINACIÓN:}

INTESTINAL: Frecuencia: Trastornos:

Medidas correctoras:

VESICAL: Frecuencia: Trastornos:

Medidas correctoras:

CUTÁNEA: Normal $\square$ Aumentada $\square$ Disminuida $\square$

DATOS DE INTERÉS: 
PATRÓN DE ACTIVIDAD-EJERCICIO:

MOVILIDAD: Autónomo $\square$ Dependiente $\square$ Ortesis $\square$ Tipo:

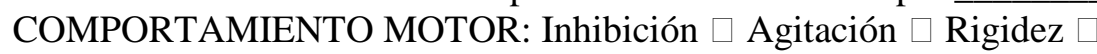

Descoordinación $\square$ Temblores $\square$ Estereotipias $\square$ Otros:

CARACTERÍSTICAS COMPORTAMIENTO MOTOR:

Controlable $\square$ Limitante $\square$ Incapacitante $\square$

ASPECTOS QUE INFLUYEN EN COMPORTAMIENTO MOTOR:

SITUACIÓN LABORAL: Activo $\square$ Desempleo $\square$ Jubilado $\square$ Pensionista $\square$

Profesión:

ACTIVIDADES DIARIAS:

ACTIVIDADES DOMÉSTICAS:

DATOS DE INTERÉS:

PATRÓN DE SUEÑO- DESCANSO:

HORAS SUEÑO NOCTURNO:___ OTROS DESCANSOS:

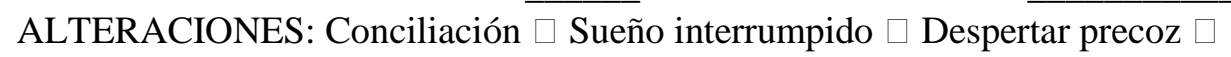

Hipersomnia $\square$ Cambio ritmo sueño $\square$ Pesadillas $\square$ Otras:

MECANISMOS PARA COMBATIR INSOMNIO:

DATOS DE INTERÉS:

PATRÓN DE AUTOPERCEPCIÓN:

SEGURIDAD EN SÍ MISMO: Si $\square$ No $\square$

AUTODESCRIPCIÓN:

AUTOESTIMA: Sobrevaloración de sus capacidades $\square$

Infravaloración de sus éxitos $\square$ Sobrevaloración de sus errores y desgracias

AUTOVALORACIÓN: Positiva $\square$ Negativa $\square$

SENTIMIENTOS RESPECTO A LA AUTOVALORACIÓN: Inferioridad

Inutilidad $\square$ Culpabilidad $\square$ Tristeza $\square$ Impotencia $\square$ Miedo $\square$ Ansiedad $\square$

Ridículo $\square$ Rabia $\square$ Superioridad $\square$

IDEACIÓN TANÁTICA: No $\square \mathrm{Si} \square$ Estructura:

DATOS DE INTERÉS:

PATRÓN COGNITIVO-PERCEPTIVO:

NIVEL DE CONCIENCIA Y ACTITUD RESPECTO AL ENTORNO:

Somnoliento $\square$ Confuso $\square$ Apático $\square$ Hipervigilante $\square$ Fluctuante $\square$

ORIENTACIÓN ESPACIAL: Si $\square$ No $\square$

ORIENTACIÓN TEMPORAL: Si $\square$ No $\square$

ORIENTACIÓN EN PERSONA: Si $\square$ No $\square$

ORGANIZACIÓN DEL CURSO DEL PENSAMIENTO:

Inhibición $\square$ Aceleración $\square$ Perseverancia $\square$ Disgregación $\square$ Incoherencia $\square$

ORGANIZACIÓN DEL CONTENIDO DEL PENSAMIENTO:

Coherente y organizado $\square$ Distorsionado $\square$ Ideación delirante $\square$

ALTERACIONES PERCEPTIVAS: Si $\square$ No $\square$

Auditivas $\square$ Visuales $\square$ Olfativas $\square$ Cenestésicas $\square$

Descripción:

EXPRESIÓN DEL LENGUAJE:

Disgregado $\square$ Verborreico $\square$ Monótono $\square$ Monosilábico $\square$ Neologismos $\square$

Otros $\square$ Especificar:

Alteraciones en el volumen: Si $\square$ No $\square$ Descripción

Alteraciones del tono y/ o la modulación: : Si $\square$ No $\square$ Descripción

DATOS DE INTERÉS: 
PATRÓN DE ROL-RELACIONES:

ESTRUCTURA FAMILIAR (FAMILIOGRAMA):

PERSONA DE SOPORTE:

LUGAR DENTRO DE LA FAMILIA Y ROL DESARROLLADO:

REACCIÓN DE LA FAMILIA ANTE LA ENFERMEDAD:

PERSONAS CON LAS QUE SE RELACIONA DIARIAMENTE:

TIPO DE RELACIÓN: Satisfactoria $\square$ Insatisfactoria $\square$ Indiferente $\square$ TIPO DE RELACIÓN CÍRCULO DE AMISTADES:

Satisfactoria $\square$ Insatisfactoria $\square$ Indiferente $\square$ No posee $\square$

TERAPIA PSICO-EDUCATIVA: Si $\square$ No $\square$ Especificar

DATOS DE INTERÉS:

\section{PATRÓN DE SEXUALIDAD-REPRODUCCIÓN:}

ACTIVIDAD SEXUAL: Satisfactoria $\square$ Insatisfactoria $\square$ No posee DISFUNCIONES SEXUALES: Si $\square$ No $\square$

CONOCIMIENTOS/ USO MEDIDAS ANTICONCEPTIVAS: Si $\square$ No $\square$

MENSTRUACIÓN: Si $\square$ No $\square$ Menopausia $\square$ Alteraciones $\square$

Especificar alteraciones:

REVISIONES GINECOLÓGICAS ANUALES: Si $\square$ No $\square$

DATOS DE INTERÉS:

PATRÓN DE ADAPTACIÓN-TOLERANCIA AL ESTRÉS:

ESTADO DE TENSIÓN/ ANSIEDAD: Generalizado $\square$ Temporal $\square$ FACTORES DESENCADENANTES: Ideas/ pensamientos $\square$ Miedos/ fobias ESTRATEGIAS DE ADAPTACIÓN Y/ O CONTROL DE LA ANSIEDAD:

Somatizaciones $\square$ Drogas/ Alcohol/ Tabaco/ Juego patológico $\square$ Inhibición $\square$ Análisis del problema $\square$ Compartir/ Comentar $\square$ Otras

Especificar:

DATOS DE INTERÉS:

\section{PATRÓN DE VALORES-CREENCIAS:}

CREENCIAS RELIGIOSAS: Si $\square$ No $\square$

Especificar:

VALORES FAMILIARES:

DATOS DE INTERÉS:

D.U.E: 
VALORACIÓN DE ENFERMERÍA AL ALTA.

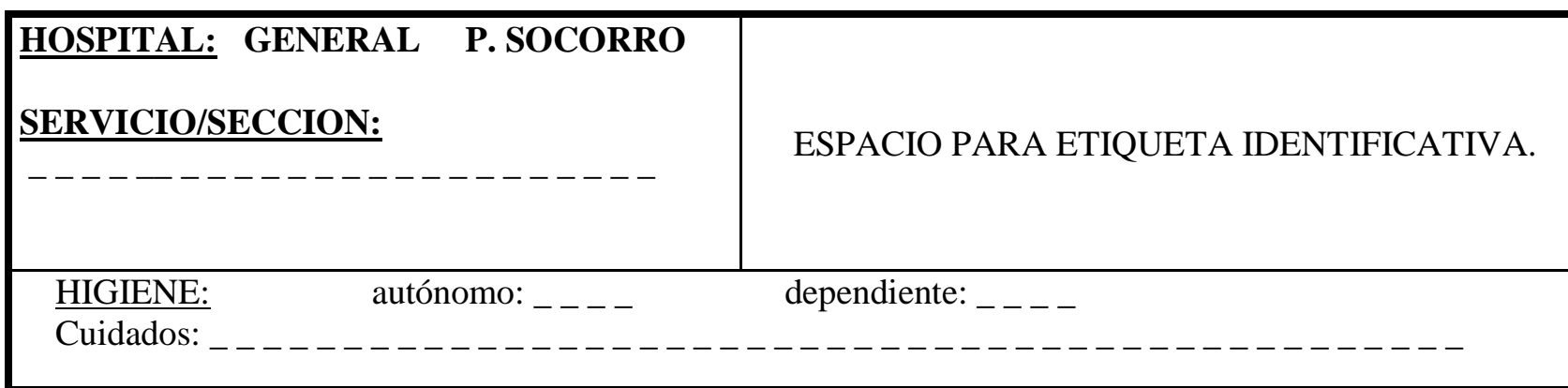

$\underline{\text { PIEL Y/O MUCOSAS: }}$

Tipo de lesión/es: úlcera: _ _ traumatismo: _ _ herida quirúrgica: _ _ otras:

Curas/cuidados:

\begin{tabular}{l}
------------------------------------------------------- \\
------------------------------------------ \\
\hline CONSCIENCIA.
\end{tabular}

CONSCIENCIA: $\quad$ orientado: $\_$desorientado: ${ }_{-}$agitado: ${ }_{-}$inconsciente: $\ldots$

MOVILIDAD: $\quad$ autónomo: _- $\quad$ dependiente: _-

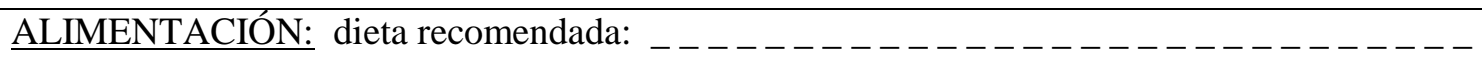

Cuidado del sondaje gástrico y/o de la gastrostomía percutánea:

OXIGENACIÓN: Horas/día: _ _ volumen: __ 1/min administración: _ _ _

Cuidados: -----------------------------------------

Traqueostomía: no de cánula: _ _ tipo:

ELIMINACIÓN:

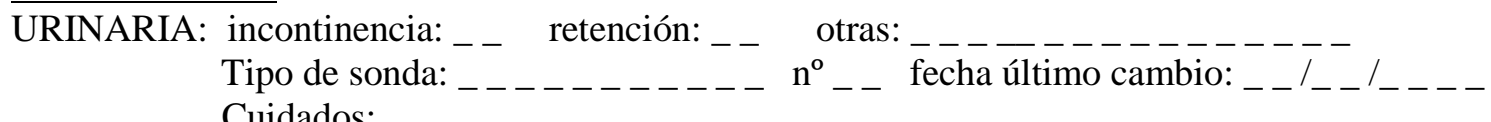

INTESTINAL: diarrea: ${ }_{-}$estreñimiento: ${ }_{-}$incontinencia: ${ }_{-}------------$

Ostomía: tipo: _ _ _ _ localización: _ _ _ _ _ estado del estoma:

Piel periestomal: _- _- _- _- efluyente:

Grado de destreza en el manejo de la ostomía:

SEGURIDAD: autónomo: _- $\quad$ dependiente: $\ldots$

\section{BIENESTAR:}

Dolor:

Estado anímico:

COMUNICACIÓN: Alteración de: visión: _ _ audición: _ _ habla:

Vías terapéuticas: tipo de catéter: __ _ $\ldots$ fecha de implantación:

Cuidados:

Observaciones/Recomendaciones al paciente y/o familiares:

-------------------------------------- - - - - - - -

Enfermera/o de referencia: Dña/ ${ }^{\circ}$

Firma:

Fecha: $----1---1---$ 
ISSN 1695-6141

@ COPYRIGHT Servicio de Publicaciones - Universidad de Murcia 